\title{
Hypervariable antigen genes in malaria have ancient roots
}

Martine M Zilversmit ${ }^{1,2,3,4^{*}}$, Ella K Chase ${ }^{3}$, Donald S Chen ${ }^{2}$, Philip Awadalla ${ }^{4}$, Karen P Day ${ }^{2}$ and Gil McVean ${ }^{3,5}$

\begin{abstract}
Background: The var genes of the human malaria parasite Plasmodium falciparum are highly polymorphic loci coding for the erythrocyte membrane proteins 1 (PfEMP1), which are responsible for the cytoaherence of $P$. falciparum infected red blood cells to the human vasculature. Cytoadhesion, coupled with differential expression of var genes, contributes to virulence and allows the parasite to establish chronic infections by evading detection from the host's immune system. Although studying genetic diversity is a major focus of recent work on the var genes, little is known about the gene family's origin and evolutionary history.

Results: Using a novel hidden Markov model-based approach and var sequences assembled from additional isolates and species, we are able to reveal elements of both the early evolution of the var genes as well as recent diversifying events. We compare sequences of the var gene DBLa domains from divergent isolates of $P$. falciparum (3D7 and HB3), and a closely-related species, Plasmodium reichenowi. We find that the gene family is equally large in $P$. reichenowi and $P$. falciparum -- with a minimum of 51 var genes in the $P$. reichenowi genome (compared to 61 in $3 D 7$ and a minimum of 48 in HB3). In addition, we are able to define large, continuous blocks of homologous sequence among $P$. falciparum and $P$. reichenowi var gene DBLa domains. These results reveal that the contemporary structure of the var gene family was present before the divergence of $P$. falciparum and $P$. reichenowi, estimated to be between 2.5 to 6 million years ago. We also reveal that recombination has played an important and traceable role in both the establishment, and the maintenance, of diversity in the sequences.

Conclusions: Despite the remarkable diversity and rapid evolution found in these loci within and among $P$. falciparum populations, the basic structure of these domains and the gene family is surprisingly old and stable. Revealing a common structure as well as conserved sequence among two species also has implications for developing new primate-parasite models for studying the pathology and immunology of falciparum malaria, and for studying the population genetics of var genes and associated virulence phenotypes.
\end{abstract}

Keywords: Non-allelic homologous recombination, Hidden Markov-model, var genes, Malaria, PfEMP1, Gene family evolution, Balancing selection

\section{Background}

Despite forty years of research, there is no effective vaccine against malaria, which caused an estimated 655,000 deaths in 2011, almost all of which are due to falciparum malaria [1]. Notably, however, people from endemic areas are able to develop natural adaptive (but not purifying) immunity by adulthood after repeated infections

\footnotetext{
* Correspondence: martine.zilversmit@nih.gov

${ }^{1}$ National Institute of Allergy of Infectious Disease, National Institutes of Health, 12735 Twinbrook Parkway, Rockville, MD 20852, USA

${ }^{2}$ Department of Microbiology, NYU Langone Medical Center, 341 East 25th Street, New York, NY 10010, USA

Full list of author information is available at the end of the article
}

[2]. One reason why the etiological agent of this form of malaria, the parasite Plasmodium falciparum, can evade the human immune system is the very high level of sequence diversity in the Plasmodium falciparum Erythrocyte Membrane Proteins 1 (PfEMP1s), expressed on the surface of infected red cells. These proteins can provoke an immune response and are known virulence factors, contributing to the adherence of infected erythrocytes to the vascular endothelium, clogging capillary vasculature in the brain and body [3]. PfEMP1s are encoded by a rapidly evolving and large multigene family (ranging from at least 40 to over 60 genes per genome), the var genes. These genes evolve extremely rapidly, to the point

\section{Biomed Central}


where they do not have stable locations in the genome [4]. Because of this mobility, combined with a very high level of sequence diversity [5], these genes cannot be described as having a conventional structure of shared alleles among populations. Recombination, combined with single-base mutation, is the mechanism of rapid evolution that generates the diversity at these loci $[4,6,7]$.

Unlike the other well-studied antigens in P. falciparum (e.g. circumsporozoite protein, the Merozoite Surface Proteins-1, -2, and -3, and Apical Membrane Antigen-1), var genes are not shared among all human malaria parasites. Only a few var genes have ever been found in another species, the closely related chimpanzee parasite $P$. reichenowi [7], suggesting that the large and complex var gene family is unique to $P$. falciparum.

A recent origin for these genes would be highly unusual given what is known about the evolution of the other important Plasmodium antigen-coding genes. Alleles at these loci in $P$. falciparum populations are frequently very old, predating the divisions of the populations and tend to be older than other loci and alleles in the genome $[8,9]$. Balancing selection acts on loci for which diversity is advantageous because of frequency dependent selection. The result of this type of selective force is that old alleles, which would typically be lost in a species or population over time, are maintained because they become more beneficial to the organism as they become rarer, hence selection drives them up in frequency again. This is the case with antigens in pathogens of vertebrates like malaria parasites, because the host's immune system is more effective against proteins it is exposed to more frequently. As a result, parasites expressing less-common proteins avoid detection more effectively.

In spite of the importance of the var genes and extensive studies of their diversity [5,10], the early evolution of the var genes has been difficult to examine because frequent recombination among paralogs renders traditional methods for examining evolution, such as phylogenetic inference, ineffective. Earlier studies have shown that mosaic recombination plays an important role in the evolution of the var2csa gene [11,12]. This gene is unique in the genome, distinct from the paralogs that code for PfEMP1s [13], and only recombines homologously. However, it is structurally related to the larger var gene family examined here. Because the var genes do not have a stable genome location or recombine in a straightforward homologous nature like the var2csa genes or other antigens in P. falciparum [8], specialized methods are required to examine their evolutionary history. Here we have developed a statistical method using a hidden Markov model to study the evolutionary relationships of the var genes and the early evolution of this complex gene family. Although these genes are known for extreme diversity and rapid recent evolution, we demonstrate an older history for this gene family than was previously shown, whereby recurrent shuffling of ancient semi-conserved sequence blocks underlies sequence diversity.

\section{Results}

var genes in Laverania: the structure of the gene family

Plasmodium falciparum and $P$. reichenowi are the only two species of the recently resurrected subgenus Laverania with available genome sequence data. Although our knowledge of the evolution and structure of the $P$. falciparum genome remains incomplete, even less is known about the genetics and genomics of $P$. reichenowi. Reconstructing the var genes in $P$. reichenowi allows for a comparative analysis and improved evolutionary understanding of these genes, which function both as virulence factors and in transmission [14].

We have annotated the available capillary sequence data of the $P$. reichenowi genome for var genes, using a prototypical sequence for the DBL $\alpha$ binding domain [15] as the model. The DBL $\alpha$ binding domain, averaging 1.8 $\mathrm{kb}$ in length, is used as the homologous region for comparison to define var genes in this study because it is the only functional domain common to all P. falciparum var gene paralogs.

Using the tBLASTx algorithm and the prototype DBL $\alpha$ domain, we were able to extract reads (typical length $900 \mathrm{bp})$ from the Sanger database [http://www.sanger.ac. $\mathrm{uk} /$ resources/downloads/protozoa/plasmodium-reichenowi. html]. Using the Clean Data assembly algorithm in Sequencher (GeneCodes), 51 unique DBL $\alpha$ regions in $P$. reichenowi were recovered (including those for the comparatively conserved var1csa and var2csa genes). This minimum number of var genes in $P$. reichenowi is within range of that for a $P$. falciparum var gene family, thus demonstrating that the family is equally large in both species. These assembled loci include some that appear to be pseudogenized by frameshifts, although this may represent sequencing errors.

Phylogenetic analysis of the var gene DBL $\alpha$ domain reveals that the $P$. reichenowi var genes do not cluster together (Figure 1). Rather, the var genes in the analysis, from $P$. reichenowi and two divergent $P$. falciparum isolates (representing the Old World (3D7), and the New World (HB3)), form a diffuse set of small groupings with very little support in the basal branches of the tree. This result indicates that the var genes likely arose as an entire family before the $P$. falciparum-P. reichenowi speciation event 2.5-6 million years ago [16]. Such an unresolved tree typically indicates rapid evolution by point mutation (saturating the informative sites), reticulate evolution by recombination such that a bifurcating 


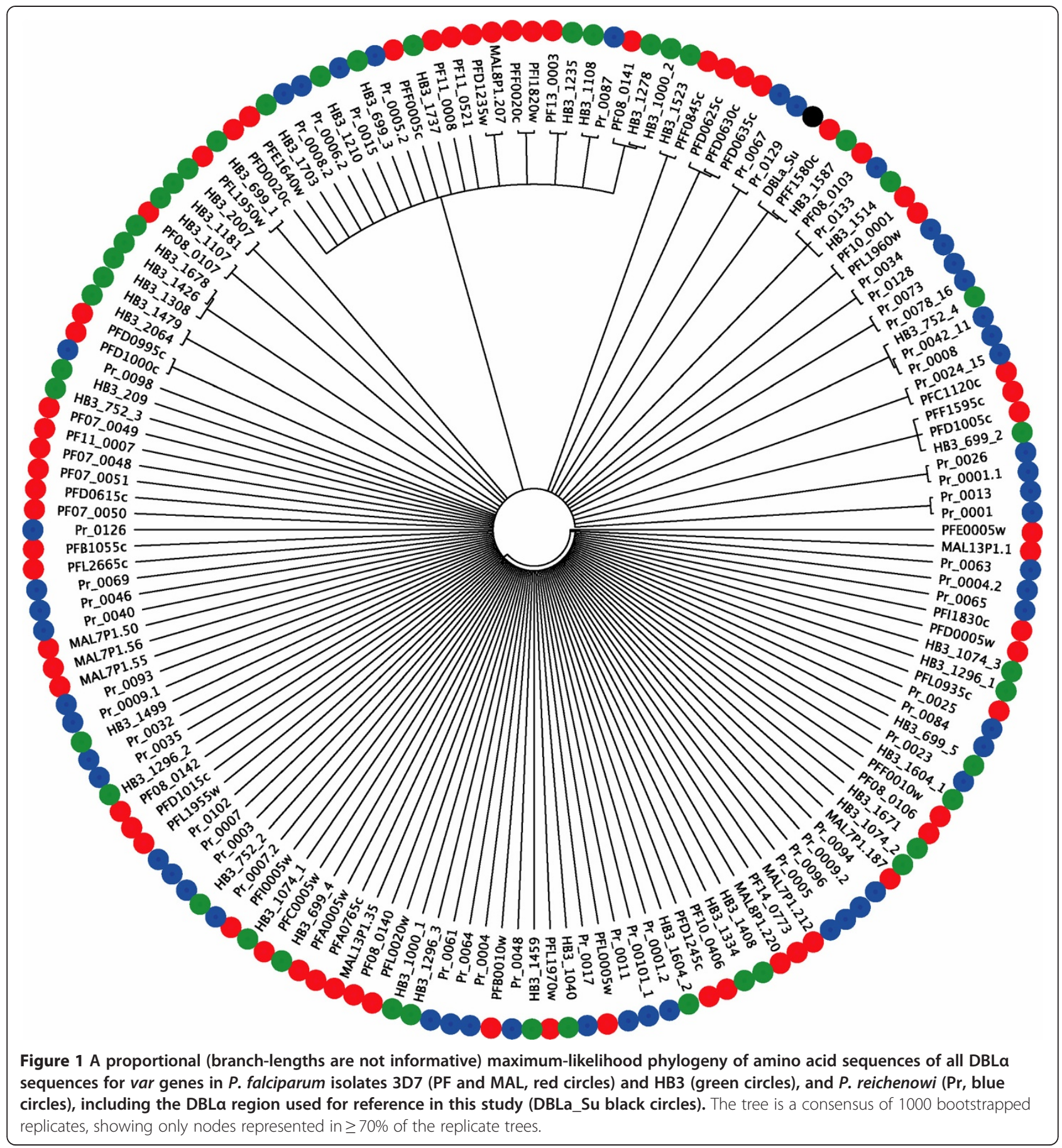

tree diagram cannot represent the evolution, or a combination of these two mechanisms.

New methods for exploring the evolution of rapidly evolving gene families

The poor performance of phylogenetic and other methods in reconstructing the history of the var genes points to the unusual evolution of these genes. Thus, new methods are necessary to identify relationships between sequences. To this end, we have developed a means to examine the homologous relationships among orthologous and paralogous sequences allowing for recombination. This statistical method, Tesserae, uses a hidden Markov model to find homology, a product of approximate conditionals (PAC) likelihood [17] to estimate the recombination parameter, a modified global alignment algorithm (Needleman-Wunsch) to detect mosaic recombination [18], and the BLOSUM62 matrix 
is used for amino acid transitions. (Figure 2A shows an example of the output of the program, see Additional file 1 for details of the method and the model). This modelbased approach aims to reconstruct each sequence in a data set as an imperfect mosaic of one or more donor sequences, allowing for substitutions, insertions, deletions, and recombination-induced mosaicism. The parameters for insertions, deletions and mutations are estimated by expectation-maximization (Baum-Welch algorithm) with no recombination (a conservative approach for detecting the effects of recombination). Subsequently, a compositelikelihood surface for the recombination parameter is calculated and, for the maximum-composite-likelihood parameter estimates, the maximum likelihood path through the HMM is calculated for each sequence (see Additional file 1). The absolute value of the likelihood, normalized for sequence length, is used as the metric by which alignment robustness is measured. The cutoff was established by training the system on known recombination breakpoints and regions known to be without recombination, discussed below. This last step is only necessary for gene families, such as the var genes, whose extremely rapid evolution makes identifying orthologous loci among individuals difficult.

The algorithm was tested using both simulated and empircal data. For simulated data we constructed amino acid sequences of 150 residues in length, in groups (gene families) of 60. Ten "gene families" were generated for each of eight sets of parameters (described in detail in the Additional file 1), with a different level of recombination and gene conversion, or insertion/deletion events, in their history. In the gene families with indels, the algorithm accurately revealed this in the estimation of an appropriately large gap extension parameter in these data, and not in data sets simulated without a history of indels. Gene families with the highest recombination rates revealed some spurious gap extension, but far less than in actual indel data (Additional file 1: Figure S2).

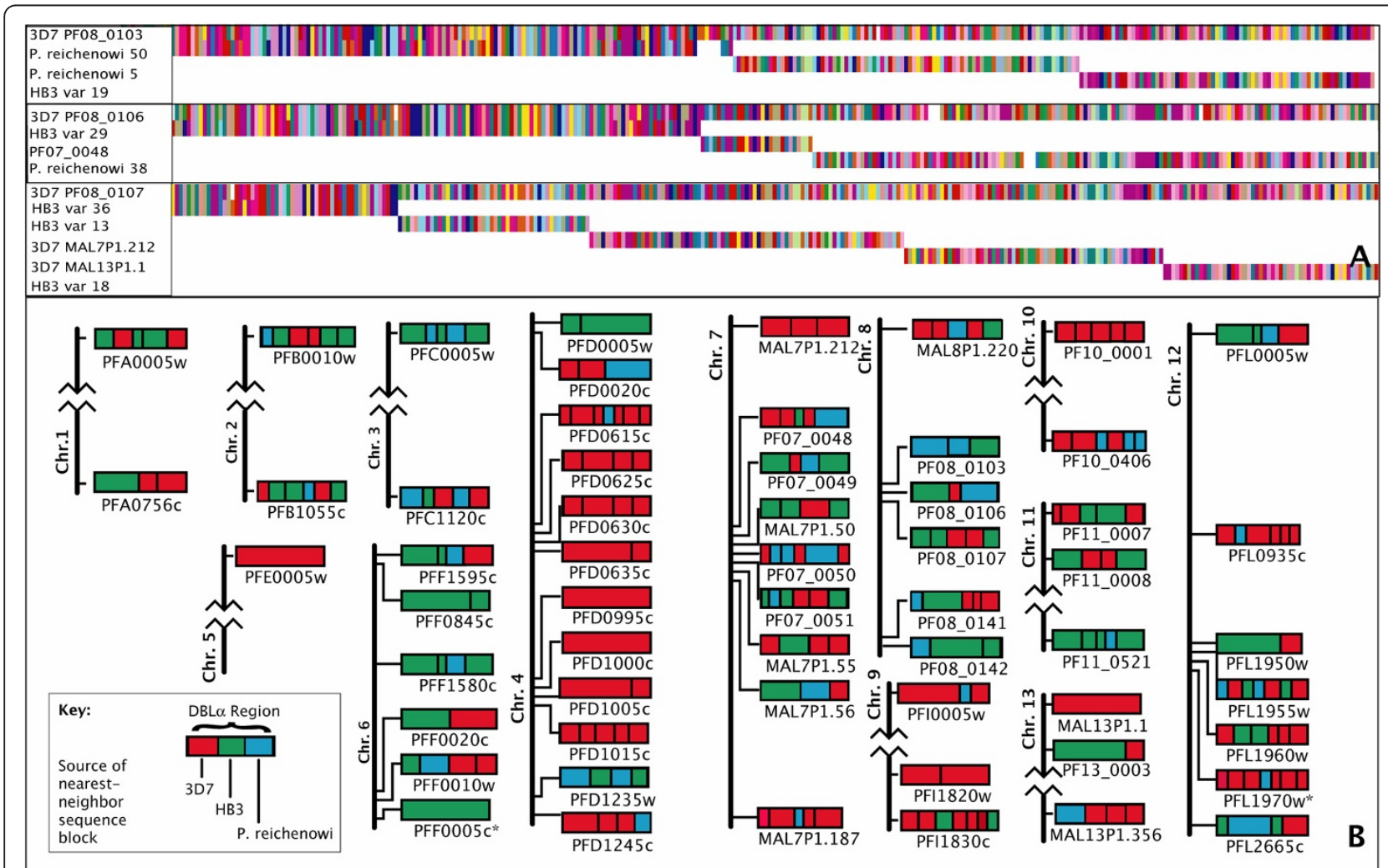

Figure 2 An example of the output and results of the analytical method. (A) Amino acid alignments are shown for three exemplary target sequences used in the analysis (the first sequence in each alignment is the target) used in the analysis. Each destination sequence is shown for the fragment (for recombinant sequences) or whole sequences (for non-recombinant results, in these examples) that showed the homology match. Each color represents a different amino acid. (B) Mosaic ancestry of the 3D7 DBLa domains. Schematic representations of domains from the 3D7 genome showing the source of the nearest-neighbor of each homology block: 3D7 (red, within genome match, recent homology), HB3 (green, within species match, older homology) and P. reichenowi (blue, across species match, ancient homology). Homology regions are separated by black vertical lines, and adjacent blocks of the same color represent blocks originating from different genes of the same genome. Genes are placed in genomic context showing relative locations to each other and to the telomeres of each chromosome (chromosomes drawn roughly to scale). Possible pseudogenes are marked with *. Only robust, high-scoring alignments are shown. Alignments for 3D7 PF08_0140 and 3D7 PFL0020w are not shown due to poor scores. 
The algorithm estimates a very low level of recombination for datasets with no recombination or indels (some bias is expected since the recombination rate must be positive), and increasingly high recombination rates for gene families simulated with comparably higher rates (Additional file 1: Figure S3). Precise calculations of the recombination rates were possible due to the controlled creation of these artificial sequences using the coalescent. Comparisons of the exact values to those calculated by the algorithm showed a high level of accuracy in the estimated recombination rates (see Additional file 1 for a detailed description of the simulation protocols and results). In addition, the difference in likelihood between models with and without recombination for each sequence was also assessed, with the statistical significance of the improvement of recombination models over those without as $\mathrm{P}<1 \times 10^{-32}$.

The algorithm was also tested against empirical recombining and non-recombining data, both for reciprocal exchange (in the B and C group var genes [19]) and gene conversion (the Acyl CoA-Synthetase gene family [20] and the var genes (PFD0625c/PDF0635c and PFD0630c/PFD0635c [21])). To test for false positives, we used a non-recombining data set, 96 complete mitochondrial genomes from P. falciparum [22]. The program was able to accurately recover all known recombination events, while finding no recombination history in the mitochondrial data, as expected.

Using Tesserae with sequence data from across individuals (or isolates in the case of pathogens) and species, it is possible to infer the relative rates of different evolutionary processes, because the source of the donor sequences reveals the relative age of the recombination events or the homologous sequence. Donors from the same genome indicate recent events, those from a divergent individual of the same species reveal events occurring since speciation, and between-species matches represent ancient homology and old recombination events.

With Tesserae, we find high-scoring alignments for all but a small minority of loci, revealing aspects of the evolutionary history of each var locus used in the analysis. Results for the DBL $\alpha$ domains in 3D7 are shown (Figure 2B). These results reveal extensive mosaic recombination at the majority of loci.

Although some within-genome, and thus recent, gene duplicates are recovered using this method (e.g. PFD0995C and PFD1000c on chromosome 4, Figure 2B), older mosaicism is widespread, with 31 of the 58 loci showing sequence that is conserved between species, in 39 sequence blocks. Both $P$. falciparum clones show $13.3 \%$ of the sequence blocks preferentially matched outside of the species, with $P$. reichenowi (Table 1 ). In addition, roughly $30 \%$ of the sequence of each isolate's
Table 1 Percent recombination blocks shared among genomes

\begin{tabular}{llll}
\hline & P. reichenowi & 3D7 & HB3 \\
\hline P. reichenowi & 52.2 & 26.7 & 21.1 \\
3D7 & 13.3 & 37.6 & 49.0 \\
HB3 & 13.3 & 60.8 & 25.9 \\
\hline
\end{tabular}

DBL $\alpha$ sequence blocks shows preferential matching within the genome itself (self-matching) (Table 1).

To examine whether the cross-species matches represented older recombination and conserved sequence, or an introgression through recent recombination, we ran the analysis with three additional sets of $P$ falciparum DBL $\alpha$ domains from three Old World isolates, that have diverged from 3D7 after speciation (Dd2 (Lao/Indochina), IGH (India), and "PfClin" (Ghana) [23]. If the $P$. reichenowi sequence that matched $3 \mathrm{D} 7$ represented a recent introgression and subsequent recombination, then these homology regions would remain the best match, even with more recently diverged sequence present (the matching between species would not be reduced in frequency). However, if the conserved sequence blocks represent recombination occurring before speciation, the recombination blocks in the DBL $\alpha$ domains of 3D7 would preferentially match with the other Old World isolates, which had separated more recently and therefore would have a higher degree of similarity. Running the analysis with the additional sequence options for matching, 11 loci in $3 \mathrm{D} 7$ have best matches to $P$. reichenowi sequences in 15 blocks (rather than 31 loci in 39 blocks, without these alternate sequences present), with $6.5 \%$ of the sequence blocks matching outside the species now ( $5 \%$ for HB3), reduced from $13.3 \%$.

In further examination of the $P$. reichenowi and $P$. falciparum DBL $\alpha$ domain homology regions, we found multiple regions of particularly high homology, classified here in two groups: core motifs and conserved peptides. The core motifs are defined as regions of $100 \%$ sequence identity that are found in at least one $P$. reichenowi gene matching two or more from P. falciparum and correspond to one of the five known regions of high homology that characterize the DBL domains (termed "homology blocks" by Rask et al. [23]). These regions are numbered HB1-5 and are between 18 and 28 residues in length [23]. We recovered 18 sequences corresponding to the conserved motifs, between 10 and 29 residues long, in 36 different $P$. reichenowi var genes and shared with 85 of those from 3D7 and HB3 (details of all loci with each identical motif are available as Additional files 2 and 3). The HB2 motifs are the most frequently represented in this group, and found in approximately half of all loci. The HB3 motifs are found in two different forms in about a third of the DBL $\alpha$ domains, and HB5 are in 
about one fifth. The HB4 motif had only one identical form match two $P$. falciparum domains (one each in 3D7 and HB3), and the HB1 motifs were not present in these results.

In addition to these core motifs, we also found 53 regions that we describe as conserved peptides, determined by pairwise matches of high identity/similarity between $P$. reichenowi and $P$. falciparum DBL $\alpha$ domains. These peptides range between 24 and 140 residues long, and between $70 \%$ and $100 \%$ identity (identical amino acids) and $80 \%$ to $100 \%$ similarity (amino acids with the same or similar functional characteristics). The conserved regions are drawn from 48 non-redundant matches, with the five additional peptides each drawn from a smaller portion of a conserved peptide, with even higher identity. While many $P$. reichenowi loci revealed none of this highly conserved sequence, some loci were represented in multiple matches, the most frequent being Preich_004 with 6 non-redundant regions plus one additional sub-region of high $P$. reichenowi/P. falciparum homology. (The full sequences of all the $P$. reichenowi core motifs and conserved peptides, plus the alignments and matching loci from P. falciparum are included in Additional files 2, 3, and 4).

Our results reveal that recombination of homology regions is shaping the var gene repertoires in the same way within and among species. This recombination, however, does not reveal a particular hot- or coldspot structure along the DBL $\alpha$ domains. Table 2 and Figure 3 show that the average distance between breakpoints, and the variance of these distances, is the same in all samples studied. The high variance in block size also reveals the lack of hot- or coldspots of recombination in the DBL $\alpha$ regions. The homogeneity of recombination rate is also evident in a mapping of the positions and frequency of recombination breakpoints along the protein sequence in an alignment, showing recombination breaks at almost every residue (Figure 4). Due to insertions and deletions within the DBL $\alpha$ domains, mapping on an

Table 2 Size and number of recombination blocks in each sample

\begin{tabular}{lll}
\hline Homology regions & Block length & No. of blocks \\
\hline P. reichenowi & \\
Mean & 76.23 & 4.00 \\
SD & 55.95 & 1.40 \\
HB3 & & \\
Mean & 77.75 & 3.98 \\
SD & 64.79 & 1.89 \\
3D7 & & \\
Mean & 76.77 & 4.05 \\
SD & 61.04 & 1.77 \\
\hline
\end{tabular}

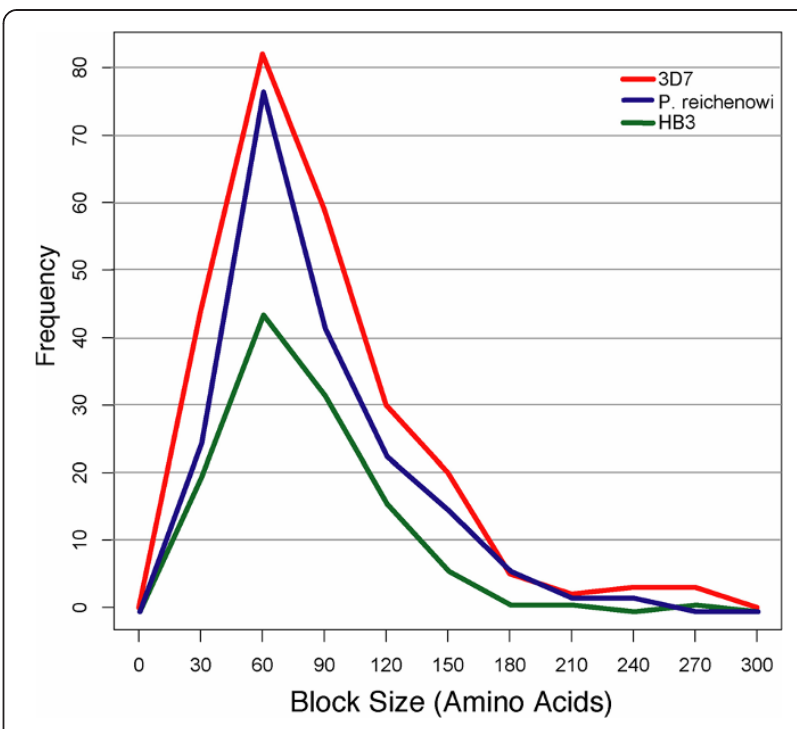

Figure 3 Distribution of recombination-block sizes. Both species and all three genomes explored in this study have the same range and mean (11) for distances between recombination breakpoints, which define recombination blocks.

alignment is necessary to ensure homologous positions are used. These insertions and deletions are in lowhomology portions of the domain and form large, gapped regions in the alignment between areas of high homology. Correcting for these gapped regions in the alignment shows that the frequency of recombination largely reflects only the number of homologous sequences (or gaps) at that position (Figure 4).

\section{Discussion and conclusions}

The similarities in size, structural organization, and sequence diversity demonstrated here for the var genes between $P$. falciparum and $P$. reichenowi establish a new understanding of the evolutionary history of this gene family. The work presented here reveals not only the existence of homologous sequence between P. falciparum and closely-related species, but also a notable amount of contiguous sequence that is shared (including exact matches for the core motifs that define the DBL $\alpha$ domains), and preservation of the size and structure of the gene family itself. We also elucidate the unusual manner by which these genes evolve, combining recombination with point mutation and birth-death evolution, to create new loci out of older sequence blocks. These findings have implications for understanding the evolution of infectious disease including host-parasite co-evolution, and for expanding new great-ape model systems to study malaria pathology.

\section{Methods for studying hypervariable paralogs}

Previous studies have shown that there is some var gene sequence shared among $P$. falciparum and $P$. reichenowi 


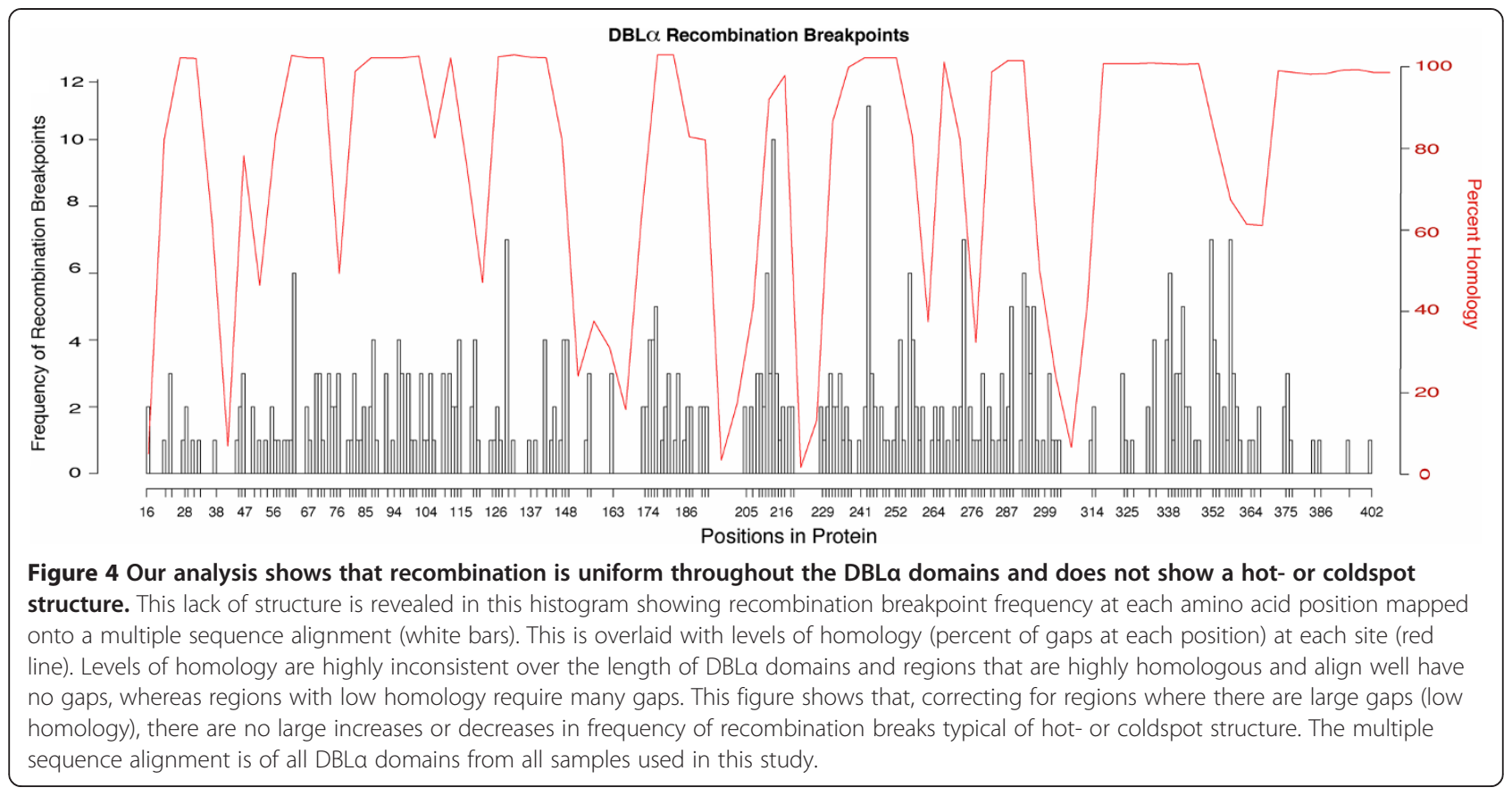

$[7,23]$, and that recombination is likely to contribute to diversifying the genes in P. falciparum [6,23]. Here we show the structure of this recombination, and large blocks of sequence, are shared between $P$. falciparum and $P$. reichenowi. We also reveal that these ancient elements are more numerous and more highly conserved than previously thought. These results suggest that the var genes are under similar balancing selection pressures evident in other antigens found among all human Plasmodium parasites (e.g. circumsporozoite protein, the Merozoite Surface Proteins-1, -2 , and -3 , and Apical Membrane Antigen-1) [9]. What is unusual about this gene family is how it is evolving. Although recombination appears to be an important mechanism for creating antigenic diversity at many loci in the $P$. falciparum genome [24] and at the var genes specifically [6], the recombination here is distinct in its high frequency, evidenced by the large number of breakpoints in the DBL $\alpha$ domains when compared to other antigenic genes [24], and by the non-allelic exchange among paralogs.

Our method of var gene recovery from the $P$. reichenowi genome has notable advantages over alternative approaches. Some var genes have likely been missed in this analysis because of the level of genome coverage and any partial genes for which the DBL $\alpha$ has been deleted will not be detected. However, we compensated for the overall low-coverage by ensuring the domains had an average of $6 \mathrm{X}$ coverage in our regions of interest. (Some loci remained at partial low-coverage, although they were long enough to show that they were unique; this is indicated in the notes of the sequences listed in the Additional file 5). This techinique has advantages over searching assembled contigs (or even genomes), or using short-read data, even at higher coverage. A starting dataset of raw shotgun reads will be less biased towards recovering loci in regions of lower variation and/or recombination, which is the problem with searching assembled contigs or even the genome sequences of Plasmodium parasites available at this time. This problem arises due the difficulty in assemblying fast-evolving regions, such as the subtelomeres of each chromosome. Also, assembly of short-read data produced from next-generations sequencing, as opposed to these long-read capillary data, is problematic in regions where the units of recombination may be equal to or smaller in size than the reads themselves, requiring much higher coverage for confident assembly. Finally, data available from shotgun sequence, such as the reads we used, will reveal a much less biased set of var genes than those revealed by targeted PCR [5,7].

\section{How var genes evolve: recombination, balancing selection, and birth/death evolution}

The findings presented here build on the existing body of work aimed at understanding the structure and evolution of the var genes. These adhesive proteins are expressed on the red-cell surface and allow malariainfected cells to adhere to the tissue of the vascular endothelium [3], out of the peripheral circulation and consequently out of the reticuloendothelial system. Because the PfEMP1s are displayed on the surface of infected cells where they are detectable by the host, maintaining diversity at these loci allows the parasites to evade detection by the human immune system. 
Previous work has shown sequence homology exists between $P$. falciparum and P. reichenowi var genes [7] and results of the present study extend these findings by demonstrating the similarity in gene family size, DBL $\alpha$ domain structure, and conserved motifs in the DBL $\alpha$ domains. These shared elements show that few characteristics of these genes are unique to $P$. falciparum. By examining the functional region common to all $P$. falciparum var genes, the DBL $\alpha$ binding domain, we find that there are at least 51 var genes in $P$. reichenowi, a similar number to what would be expected from this large gene family in P. falciparum (approximately 50-60 per genome). These $P$. reichenowi DBL $\alpha$ domains retain the same structure as those identified in $P$. falciparum, with a series "homology blocks" [23] containing sequence that has remained identical between the species over evolutionary time. These results, coupled with those that show that half of the genes in a single genome reveal an element of transpecies ancestry (preferentially over intraspecific origins), shift our understanding of these genes as being composed of sequence that is solely rapidly evolving. Here we show that the unusual elements of the var genes are not the recent origins of the genes themselves or the size of the gene family, but the way they have evolved such that older sequence recombines among paralogs (non-allelic homologous recombination) to encode novel antigenic proteins. If the majority of the sequence in the DBL $\alpha$ domains was very new (fastevolving), the signal of homology among species would have been lost since the split between the human and chimpanzee parasite lineages 2.5 to 6 million years ago [16]. The maintenance of ancient diversity shared among the gene copies in divergent genomes likely points to the ongoing arms-race between the pathogen and the host's immune response. These results indicate the existence of a form of balanced polymorphism that is giving these blocks of sequence old roots relative to other loci in the genome. Under standard evolutionary forces, we expect that alleles in a population to trace their origins to the time of speciation, or subsequent events [25]. The preservation of ancient diversity, although rare for standard loci in a genome, has been found to be common for other antigenic proteins in malaria parasites $[8,9]$.

Selective pressures for increasing diversity at antigenic loci can maintain sequence morphs from common ancestral species that would normally be lost after speciation. This phenomenon occurs because, as the sequences decline in frequency over time after speciation, they become more advantageous for the individuals that carry them. This frequency-dependent selection then drives the sequence (normally alleles, but in this case the sequence blocks) back up in frequency. The host's immune system, the selective pressure in this case, is more sensitive to antigens that are more common and, as a result, parasites expressing rarer versions of a sequence have an improved chance of surviving immune detection by the host. This immune selection makes a rare sequence more beneficial.

Balancing selection of this type also has been shown to occur at immune-associated loci in vertebrates. It is notable that these immune loci are coevolving with antigenic proteins, such as the PfEMP1, in order to detect them for the vertebrate host. Trans-species alleles have been maintained at the MHC loci (critical for mammalian immunity to infections disease) of great apes over evolutionary time by balancing selection, with recombination known to be key in diversification of the human alleles [26]. Human MHC loci, when compared with those of chimpanzees, show the same trans-species mosaicism maintained by recombination that is revealed here among the var genes. In the MHC, however, this gene family recombination is happening at a notably lower frequency, with very little non-allelic exchange, and only in the loci associated with the most extreme levels of diversity [unpublished observations, Zilversmit, McVean, and Awadalla]. Therefore the maintenance of such old sequence in these two species of Plasmodium is likely due to host-parasite coevolution. It is important to distinguish the mode of evolution of the var genes, however, because it is not an entire gene, or even an entire domain, that is being maintained. Rather it is a continuous section of sequence located within the DBL $\alpha$ domain that is being maintained, which can be exchanged among paralogs as a unit by recombination.

Although recombination in clearly frequent among the DBL $\alpha$ domains, our analysis reveals no hotspot structure for the type of intradomain non-allelic homologous recombination examined, recombination at homologous loci among different paralogs. However, DBL recombination hotspots in the var genes have been found [23]. The structure of var genes consist of more than just the DBL $\alpha$ domain, rather they are a series of DBL domains (e.g. DBL $\beta$ and DBL $\gamma$ ) interspersed with CIDRs (cysteine-rich inter-domain regions). The DBL $\alpha$ domain is unique in that it is the only domain that all var genes paralogs have in common (and in the same position in the gene), however each gene has multiple different DBL domains of the other types. Hotspots do occur when interdomain DBL recombination is considered [23], e.g. between DBL $\beta$ and DBL $\gamma$, but do not appear to be present in intradomain DBL $\alpha$ recombination. The difference in the structure of recombination within domains as opposed to between them is likely due to homology. The DBL $\alpha$ domains are orthologous (homologous due to common ancestry) in this context and have more sequence similarity throughout than an interdomain comparison where the relationship among the domains is more like that of paralogs. As a result, the paralogs 
have more unevenly distributed regions of higher similarity among each other and these portions of sequence are more likely to be nucleation points for recombination breaks.

The results presented here show that the var genes are evolving through a combination of birth-death evolution, point mutation, and rapid and unstructured mosaic recombination. The point mutation is evident in the regions of high amino acid similarity, because these regions diversify by changing codons and thus the amino acid identity, while likely retaining much if not all of the function of the regions. This may allow for a level of sequence diversity that can evade immune recognition without large changes to the protein structure [27]. Birth-death evolution is a common mechanism of gene family evolution, characterized by the continual duplication and deletion of paralogs through unequal crossing over during recombination, evidenced in the var gene family by the variability in the paralog number among isolates, and in the apparent recent duplications revealed in the self-genome matches of some DBL $\alpha$ domains in this analysis (e.g. PFD0995c and PFD1000c on chromosome 4, Figure 2B).

\section{Future directions: population genetics and new primate models of malaria}

To fully explore the impact of this new understanding of the var genes, detailed comparative studies are necessary comparing the genomes of other species closely related to P. falciparum, as well as examining whether the same evolutionary dynamics are evident at the populationlevel. Multiple new species of malaria parasites have been recently discovered to be closely related to $P$. falciparum, notably P. gaboni [28] and an estimated 10 other new Plasmodium species of Great Apes [16,29] grouped into the resurrected subgenus Laverania. Our results here reveal that many of these new species are likely to have a full suite of var genes, because these parasites are shown to be more closely related to $P$. falciparum than $P$. reichenowi. At what point these genes arose in the Laverania lineage is an important evolutionary question that can only be addressed by comparative genomics among these new species.

This study was designed to examine the early evolution of the var genes. The same methods can be extended to examine the influence of recombination and the balanced maintenance of ancient diversity in their recent evolution. These studies can be accomplished by looking at microevolutionary patterns using populationlevel data. Although there is an abundance of data on var genes from field isolates $[5,10]$, these data remain largely unexplored because the correct tools to disentangle the complex histories of these genes, such as the Tesserae program, did not exist. It is possible that by identifying highly conserved regions in field isolates, scientists may be able to trace the frequency with which var genes recombine in nature, examine correlations of conserved markers with virulence phenotypes in the parasites, and explore the migration of new paralogs the way drug resistance alleles have been tracked from their originating populations as they spread throughout the world.

\section{Methods \\ DBLa sequence assembly \\ $P$. reichenowi}

DNA sequences for DBL $\alpha$ domains were assembled using approximately 900 reads and contigs from the CDC1 sample of $P$. reichenowi from the Wellcome Trust Sanger Institute [http://www.sanger.ac.uk/resources/ downloads/protozoa/plasmodium-reichenowi.html]. These reads were retrieved with the tBLASTx algorithm using the DBL $\alpha$ domain from Su et al. 1995 [15] as a reference. The reads and contigs were then assembled and edited using Sequencher v.4.2.2 software (Gene Codes Corporation, $\mathrm{MI})$, with at least $6 \mathrm{X}$ coverage necessary to call a region (although some DBL $\alpha$ regions with partial low coverage were used if the regions proved to be unique). This method retrieved some DBLß domains as well, these were not used in the analysis, but ensured that an exhaustive search for DBL $\alpha$ domains was completed.

The DNA sequences were then converted to protein using MacClade software version 4.06 [30]. Additional alignments were generated using CLUSTALW2 software [31] with a BLOSUM30 matrix, a gap opening penalty of 10.0, and gap extension penalty of 2.0 were used to filter for redundant domains.

\section{P. falciparum}

DBL $\alpha$ domains were identified using CLUSTALW2 alignments as above from whole var gene sequences using the domain from Su et al. 1995 [15] as a reference. These whole gene protein sequences were retrieved from PlasmoDB version 4.0 (for the isolate 3D7) and from sequence referenced in S. M. Kraemer et al., 2007 [4], supplied by the authors (for HB3). The whole var gene sequences were edited to the DBL $\alpha$ domains using MacClade software version 4.06 [31].

\section{Phylogenetic analysis}

All DBL $\alpha$ amino acid sequences in this study were aligned using MUSCLE [32] in Full mode using 16 iterations. The phylogeny was constructed using PhyML [33] with the LG [34] model, NNI tree topology search, and a BioNJ starting tree. 1000 trees were made with bootstrapped data and a consensus tree of all nodes appearing in $70 \%$ or more of these trees was made using Phyutility [35]. 


\section{The Tesserae program}

The Tesserae program implementing the HMM is written in $\mathrm{C}$ language and is available from the authors on request along with a document on how to install and use the program.

\section{Additional files}

\section{Additional file 1: Hidden Markov-Model to Recover Mosaic} Recombination. A PDF file showing the full hidden Markov model, in detail, and added information on tests using simulated data.

Additional file 2: Analysis and Results of Core Motifs and Conserved Peptides. An MS Word document describing and summarizing the analysis of core motifs and conserved peptides between $P$. reichenowi and $P$. falciparum.

Additional file 3: Core motifs (conserved motif classification) and genes with each one. Core motifs ordered alphabetically, with the listing of all loci with that motif.

Additional file 4: Alignments of conserved peptides. An MS Word document showing all alignments between $P$. reichenowi and $P$. falciparum for conserved peptides.

Additional file 5: Plasmodium reichenowi DBLa Domains. A tab delimited file containing curated Plasmodium reichenowi DBLa domains used in this research.

\section{Competing interests}

The authors have no competing interests.

\section{Authors' contributions}

$M M Z, G M$, and KPD conceived of the original concept. MMZ and GM conceived of and carried out the experiments and prepared the manuscript. EKC and GM constructed the hidden Markov model. DSC contributed to the analysis, experimental concept, and manuscript preparation. KPD and PA contributed to the experimental concept and manuscript preparation. All authors read and approved the final manuscript.

\section{Acknowledgements}

The work in this paper would not be possible without the free availability of unpublished data from the Wellcome Trust Sanger Institute. Additionally, we would like to thank Dr. Sue Kyes for access to HB3 var gene sequences, to Dr. James Cotton for helpful discussion on the var gene DBLa phylogeny, Dr. Jane Carlton for helpful discussion on gene family evolution, to Dr. Sebastian Gurevich for aid in manuscript preparation, and two anonymous reviewers for helpful comments. This work was supported by a Royal Society North American Visiting Scholar Fellowship (MMZ and GM), a National Institutes of Health Ruth L. Kirschstein National Research Service Award (F32Al071765, DSC), an EPSRC studentship (EKC), a Human Frontiers in Science Research Grant (PA and GM) and a Wellcome Trust Program Grant Award WT041354 (KD) to and NIH NIAID 1R01AI084156 (KD and DSC).

\section{Author details}

${ }^{1}$ National Institute of Allergy of Infectious Disease, National Institutes of Health, 12735 Twinbrook Parkway, Rockville, MD 20852, USA. ²Department of Microbiology, NYU Langone Medical Center, 341 East 25th Street, New York, NY 10010, USA. ${ }^{3}$ Department of Statistics, University of Oxford, 1 South Parks Road, Oxford OX1 3TG, UK. ${ }^{4} \mathrm{CHU}$ Sainte-Justine Centre de Recherche, Universit de Montral, 3175 Cote-Ste-Catherine, Montreal, QC, H3T 1C5, Canada. ${ }^{5}$ Wellcome Trust Centre for Human Genetics, Roosevelt Drive, Oxford OX3 7BN, UK.

Received: 13 August 2012 Accepted: 6 May 2013

Published: 31 May 2013

\section{References}

1. WHO: World Health Organization World Malaria Report. 2011. http://www.who.int/malaria/world_malaria_report_2011/en/.
2. Gupta S, Day KP: A theoretical framework for the immunoepidemiology of Plasmodium falciparum malaria. Parasite Immunol 1994, 16(7):361-370.

3. Chen Q, Schlichtherle M, Wahlgren M: Molecular Aspects of Severe Malaria. Clin Microbiol Rev 2000, 13(3):439-450.

4. Kraemer SM, Kyes SA, Aggarwal G, Springer AL, Nelson SO, Christodoulou Z, Smith LM, Wang W, Levin E, Newbold Cl, et al: Patterns of gene recombination shape var gene repertoires in Plasmodium falciparum: comparisons of geographically diverse isolates. BMC Genomics 2007, 8:45.

5. Barry AE, Leliwa-Sytek A, Tavul L, Imrie H, Migot-Nabias F, Brown SM, McVean GAV, Day KP: Population Genomics of the Immune Evasion (var) Genes of Plasmodium falciparum. PLoS Pathog 2007, 3(3):e34.

6. Freitas-Junior LH, Bottius E, Pirrit LA, Deitsch KW, Scheidig C, Guinet F, Nehrbass U, Wellems TE, Scherf A: Frequent ectopic recombination of virulence factor genes in telomeric chromosome clusters of $\mathrm{P}$. falciparum. Nature 2000, 407(6807):1018-1022.

7. Bull PC, Buckee CO, Kyes S, Kortok MM, Thathy V, Guyah B, Stoute JA, Newbold Cl, Marsh K: Plasmodium falciparum antigenic variation. Mapping mosaic var gene sequences onto a network of shared, highly polymorphic sequence blocks. Mol Microbiol 2008, 68(6):1519-1534.

8. Ferreira MU, Zilversmit M, Wunderlich G: Origins and evolution of antigenic diversity in malaria parasites. Curr Mol Med 2007, 7(6):588-602.

9. Roy SW, Ferreira MU, Hartl DL: Evolution of allelic dimorphism in malarial surface antigens. Heredity 2006, 100(2):103-110.

10. Lavstsen T, Salanti A, Jensen ATR, Theander TG, Copenhagen D: Subgrouping of Plasmodium falciparum 3D7 var genes based on sequence analysis of coding and non-coding regions. Malar J 2003, 2:27.

11. Trimnell AR, Kraemer SM, Mukherjee S, Phippard DJ, Janes JH, Flamoe E, Su XZ, Awadalla P, Smith JD: Global genetic diversity and evolution of var genes associated with placental and severe childhood malaria. Mol Biochem Parasitol 2006, 148(2):169-180.

12. Bockhorst J, Lu F, Janes JH, Keebler J, Gamain B, Awadalla P, Su X-Z, Samudrala R, Jojic N, Smith JD: Structural polymorphism and diversifying selection on the pregnancy malaria vaccine candidate VAR2CSA. Mol Biochem Parasitol 2007, 155(2):103-112.

13. Salanti A, Staalsoe T, Lavstsen T, Jensen ATR, Sowa MPK, Arnot DE, Hviid L, Theander TG: Selective upregulation of a single distinctly structured var gene in chondroitin sulphate A-adhering Plasmodium falciparum involved in pregnancy-associated malaria. Mol Microbiol 2003, 49(1):179-191.

14. Chen DS, Barry AE, Leliwa-Sytek A, Smith TA, Peterson I, Brown SM, MigotNabias F, Deloron P, Kortok MM, Marsh K: A molecular epidemiological study of var gene diversity to characterize the reservoir of Plasmodium falciparum in humans in Africa. PLOS One 2011, 6(2):e16629.

15. Su X, Heatwole VM, Wertheimer SP, Guinet F, Herrfeldt JA, Peterson DS, Ravetch JA, Wellems TE: The large diverse gene family var encodes proteins involved in cytoadherence and antigenic variation of Plasmodium falciparum-infected erythrocytes. Cell 1995, 82(1):89-100.

16. Liu W, Li Y, Learn GH, Rudicell RS, Robertson JD, Keele BF, Ndjango J-BN, Sanz CM, Morgan DB, Locatelli S, et al: Origin of the human malaria parasite Plasmodium falciparum in gorillas. Nature 2010 467(7314):420-425

17. Li N, Stephens M: Modeling Linkage Disequilibrium and Identifying Recombination Hotspots Using Single-Nucleotide Polymorphism Data. Genetics 2003, 165:2213-2233.

18. Durbin R, Eddy SR, Krogh A, Michison G: Biological sequence analysis: probabilistic models of proteins and nucleic acids. Cambridge, UK: Cambridge University Press; 1998.

19. DePristo MA, Zilversmit MM, Hartl DL: On the abundance, amino acid composition, and evolutionary dynamics of low-complexity regions in proteins. Gene 2006, 378:19-30.

20. Bethke LL, Zilversmit M, Nielsen K, Daily J, Volkman SK, Ndiaye D, Lozovsky ER, Hartl DL, Wirth DF: Duplication, gene conversion, and genetic diversity in the species-specific acyl-CoA synthetase gene family of Plasmodium falciparum. Mol Biochem Parasito/ 2006, 150(1):10.

21. Flick K, Chen Q: var genes, PfEMP1 and the human host. Mol Biochem Parasitol 2004, 134(1):3-9.

22. Joy DA, Feng X, Mu J, Furuya T, Chotivanich K, Krettli AU, Ho M, Wang A,

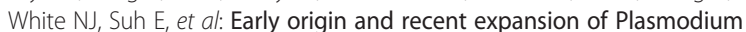
falciparum. Science 2003, 300(5617):318-321.

23. Rask TS, Hansen DA, Theander TG, Gorm Pedersen A, Lavstsen T: Plasmodium falciparum Erythrocyte Membrane Protein 1 Diversity in 
Seven Genomes: Divide and Conquer. PLoS Comput Biol 2010, 6(9):e1000933.

24. Zilversmit MM, Volkman SK, DePristo MA, Wirth DF, Awadalla P, Hartl DL: Low-complexity regions in Plasmodium falciparum: missing links in the evolution of an extreme genome. Mol Biol Evol 2010, 27(9):2198-2209.

25. Hartl DL, Clark AG: Principles of Population Genetics, 4th Edition. 4th edition. Sunderland, MA: Sinauer Associates, Inc.; 2007.

26. Parham P, Ohta T: Population Biology of Antigen Presentation by MHC Class I Molecules. Science 1996, 272(5258):67.

27. Gillbert SC, Plebanski M, Gupta S, Morris J, Cox M, Aidoo M, Kwiatkowski D, Greenwood BM, Whittle HC, Hill AVS: Association of Malaria Parasite Population Structure, HLA, and Immunological Antagonism. Science 1998, 279(5354):1173-1177.

28. Ollomo B, Durand P, Prugnolle F, Douzery E, Arnathau C, Nkoghe D, Leroy E, Renaud F: A New Malaria Agent in African Hominids. PLoS Pathog 2009, 5(5):e1000446.

29. Zilversmit MM, Awadalla P: The Importance of the Natural History of Malaria (E-Letter). In Science. 2011. vol. http://www.sciencemag.org/content/ 329/5999/1586/reply.

30. Maddison DR, Maddison WP: MacClade 4: Analysis of Phylogeny and Character Evolution. MA, USA: Sinauer Associates, Sunderland; 2000.

31. Larkin MA, Blackshields G, Brown NP, Chenna R, McGettigan PA, McWilliam H, Valentin F, Wallace IM, Wilm A, Lopez R: Clustal W and Clustal X version 2.0. Bioinformatics 2007, 23(21):2947.

32. Edgar RC: MUSCLE: multiple sequence alignment with high accuracy and high throughput. Nucleic Acids Res 2004, 32(5):1792-1797.

33. Guindon S, Dufayard JF, Lefort V, Anisimova M, Hordijk W, Gascuel O: New algorithms and methods to estimate maximum-likelihood phylogenies: assessing the performance of PhyML 3.0. Syst Biol 2010, 59(3):307-321.

34. Le SQ, Gascuel O: An improved general amino acid replacement matrix. Mol Biol Evol 2008, 25(7):1307-1320.

35. Smith SA, Dunn CW: Phyutility: a phyloinformatics tool for trees, alignments and molecular data. Bioinformatics 2008, 24(5):715-716.

doi:10.1186/1471-2148-13-110

Cite this article as: Zilversmit et al.: Hypervariable antigen genes in malaria have ancient roots. BMC Evolutionary Biology 2013 13:110.

\section{Submit your next manuscript to BioMed Central and take full advantage of:}

- Convenient online submission

- Thorough peer review

- No space constraints or color figure charges

- Immediate publication on acceptance

- Inclusion in PubMed, CAS, Scopus and Google Scholar

- Research which is freely available for redistribution

Submit your manuscript at www.biomedcentral.com/submit 